\title{
Report
}

\section{Power System Design Compromises for Large-Scale Linear Particle Accelerators}

\author{
K. Papastergiou, D. Aguglia
}

Keywords: accelerators, system integration, power supply, multilevel converter, protection device

\begin{abstract}
This paper discusses various design aspects of a 280MW Power System for the Compact LinearCollider (CLIC), a 50km long electrons-positrons accelerator, under feasibility evaluation. The key requirements are a very high accelerator availability and constant power flow from the utility grid, considering the pulsed power nature of CLIC. Firstly, the possible power network and cabling layouts are discussed along with potential difficulties on electrical fault clearance. Following, the use of active front-end converters is examined as a means to control the power flow and power quality seen by the $400 \mathrm{kV}$ grid. In particular a modular multilevel converter preliminary configuration is described and the compromises related to energy storage and voltage level are discussed.
\end{abstract}

Presented at:

EPE

Lille, France

September, 2013 


\title{
Power System Design Compromises for Large-Scale Linear Particle Accelerators
}

\author{
Konstantinos D. Papastergiou and Davide Aguglia \\ CERN - European Organisation for Nuclear Research \\ 1211 Geneva, Switzerland - k.papastergiou@cern.ch
}

\section{Keywords}

«accelerators», «system integration», «power supply», «multilevel converters», «protection device»

\begin{abstract}
This paper discusses various design aspects of a 280MW Power System for the Compact Linear Collider (CLIC), a 50km long electrons-positrons accelerator, under feasibility evaluation. The key requirements are a very high accelerator availability and constant power flow from the utility grid, considering the pulsed power nature of CLIC. Firstly, the possible power network and cabling layouts are discussed along with potential difficulties on electrical fault clearance. Following, the use of active front-end converters is examined as a means to control the power flow and power quality seen by the $400 \mathrm{kV}$ grid. In particular a modular multilevel converter preliminary configuration is described and the compromises related to energy storage and voltage level are discussed.
\end{abstract}

\section{Introduction}

The technological feasibility of a new linear particles accelerator, the Compact Linear Collider (CLIC), is under evaluation at the European Organization for Nuclear Research (CERN) [1]. CLIC aims to alleviate the effects of Synchrotron Radiation emitted by electrons and positrons (antielectrons particle) when subject to radial acceleration in circular machines such as synchrotrons. Synchrotron radiation implies a particle energy loss, which can be avoided in a linear accelerator where particle beam can reach higher energies. The main CLIC concept is illustrated in Fig. 1 (right), where the layout is composed of a main beam accelerator, which is fed by Radio Frequency (RF) power source, the so called drive beam. From the electrical engineer point of view this corresponds to an accelerator Darlington concept (like for transistors). To accelerate the particles of the drive beam a total of $280 \mathrm{MW}$ of average power is necessary to feed the $\sim 1600$ klystron modulators required for RF power production.
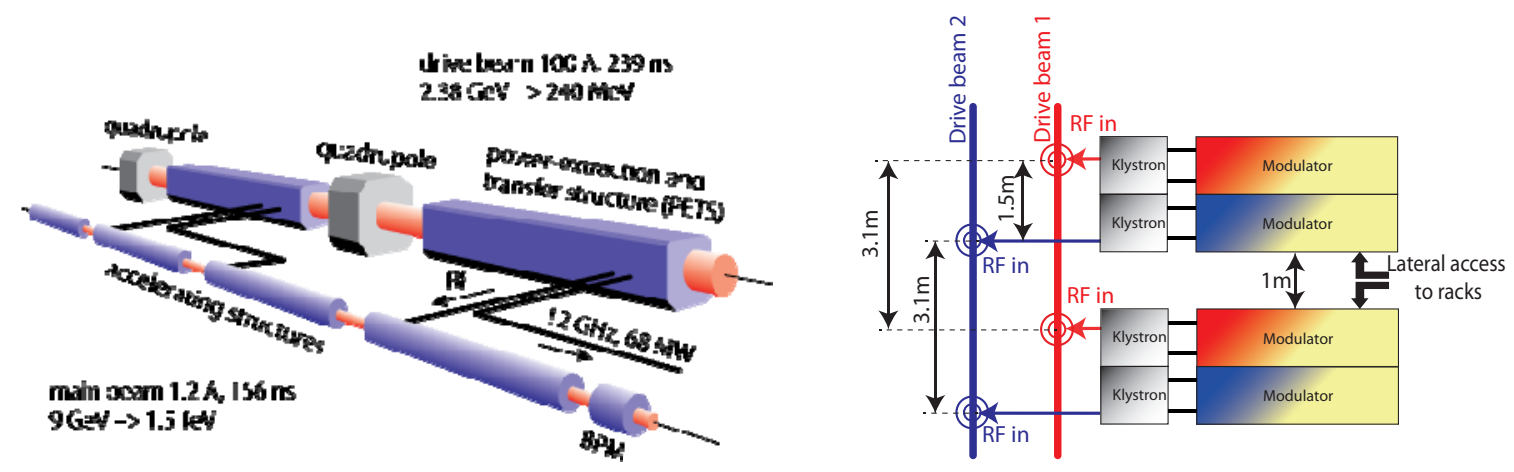

Fig. 1: Left: Energy transfer principle from drive beam to main beam; right: Klystron modulator layout for the drive beam accelerator. 
In CLIC the acceleration power is not continuously required and klystron modulators operate in pulsed mode to feed the klystron with voltage pulses of $160 \mathrm{kV}$ and $140 \mu \mathrm{s}$ length, with a repetition rate of $50 \mathrm{~Hz}$. The peak power per pulse, per klystron modulator, is $24 \mathrm{MW}$. The drive beam complex is $\sim 2.5 \mathrm{~km}$ long and a possible klystron modulator layout is presented in Fig. 1 (right). One of the biggest challenges for the power system design lies in the requirement of constant power consumption from the utility grid, which can certainly not accept a $24 \mathrm{MWx} 1600=38 \mathrm{GW}$ of power fluctuation.

The paper discusses the key aspects of a 280MW Power System for the Compact Linear Collider (CLIC). The magnitude of pulsed power required for such applications introduces a number of challenges regarding the architecture of the power system as well as the interconnection to existing power systems. Designing power electronic converters that could effectively match the transient operation of the collider is the main objective. Power distribution within the accelerator complex is also challenging due to the large distances and the parasitic elements of the distribution cabling. Modularity is a key aspect to be considered in the power system in order to reach very high accelerator availability in case of power electronics components failures. A modular Active Front-End (AFE) design is essential for decreasing the number of interventions, down-times and spare parts cost. Therefore, the application of Modular Multilevel Converters (MMC) as an interfacing grid AFE is examined and suitability in pulsed operation is preliminary examined.

\section{CLIC Power System layout}

CLIC will require power transmission from a substation to every klystron modulator over a distance of a few meters to a maximum of $1.25 \mathrm{~km}$ (if a central placement of the AFEs, with respect to the drive beam complex, is considered). The actual power system layout will be based on a complex technical-economic analysis that takes into account various component specifications, parameters and costs. The average power requirement from the AC utility grid is approximately $280 \mathrm{MW}$. However, the significant current pulsation at the load side implies that a certain fluctuation of the power drawn from the AC grid could be seen, even considering that capacitor banks in klystron modulators are attenuating $80 \%$ to $90 \%$ of power fluctuation.

The above implies that power electronics along with adequate energy storage will be needed as a front-end of the CLIC power system. The possibility of implementing a number of active front-end converters each powering a group of $160 \mathrm{~kW}$ (average power) klystron modulators is illustrated in Fig. 2(a).
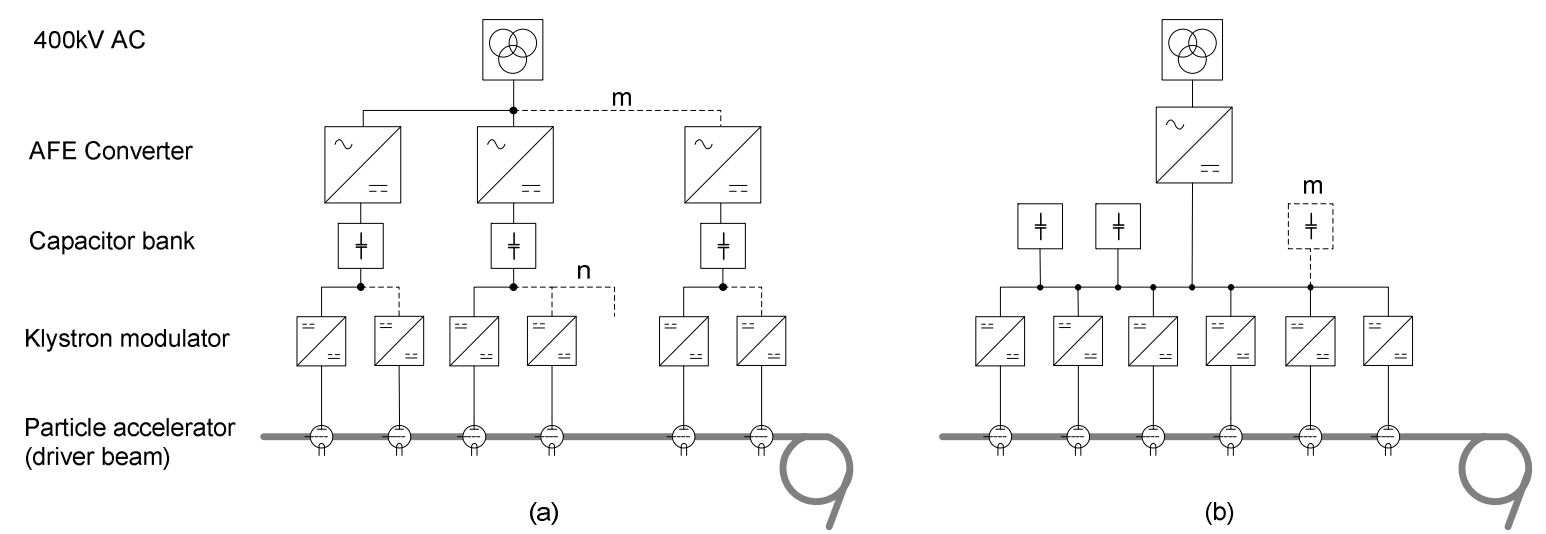

Fig. 2: Two possible configurations of the CLIC power system with (a) distributed active front-end converters supplying a group of klystron modulators and (b) centralised active front-end with medium voltage DC distribution and distributed capacitor banks 
An alternative centralised solution of a single active AC/DC converter station rated at full power could also be considered. A medium voltage DC distribution could be used to power the klystron modulators and distributed energy storage could be used to stabilise the DC voltage across the DC distribution line. The selection of the exact location and number of AFE has to be based on a global approach considering several aspects. Here the following inputs are considered:

- Current and voltage dynamics (bandwidth) required from the AFEs in order to respect klystron modulators pulse profile.

- Protection of the power system in case of short-circuit, flashover and other faults.

- Civil engineering costs affecting the location of capacitor banks, converter stations etc.

- Amount of energy storage vs. AFE current and voltage dynamics capabilities for constant power consumption from the utility grid.

The grid layout must consider a certain number of limiting factors. Particularly one has to consider the maximum manageable current and standard voltages of circuit breakers. At CERN three standard voltage levels are used:

1. $400 \mathrm{kV}$ power lines from the French grid power provider (EDF).

2. $66 \mathrm{kV}$ for distant sites interconnection at high power.

3. $18 \mathrm{kV}$ for medium distance and medium power distribution.

Standard $66 \mathrm{kV}$ and $18 \mathrm{kV}$ circuit breakers are rated at $3 \mathrm{kA}$ of nominal RMS current; with an opening short-circuit current capability of $20 \mathrm{kA}$ to $25 \mathrm{kA}$. Short-circuit current values are limited by the busbars standard mechanical construction. Considering standard circuit breakers the maximal manageable power per $400 \mathrm{kV} / 66 \mathrm{kV}$ transformer is $P_{\max 66}=\sqrt{3} \times 66000 \times 3000=343 M W$, whereas for $66 \mathrm{kV} / 18 \mathrm{kV}$ transformers the maximal nominal power would be $P_{\max 18}=93 \mathrm{MW}$. Economically, an interesting solution would be trying to group all power electronics in a minimal number of AFEs. Considering standard substation components presented above the minimum $18 \mathrm{kV}$ AFEs number would be three, as depicted in Fig. 3 3(a). Furthermore it is possible to directly step-down the voltage with $400 \mathrm{kV} / 18 \mathrm{kV}$ transformer, leading to the minimal transformers number grid configuration of Fig. $3(b)$.

a)

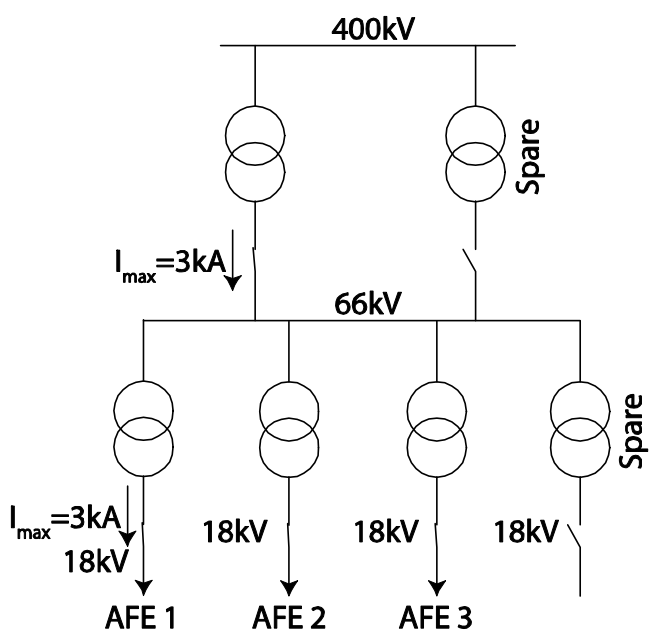

b)

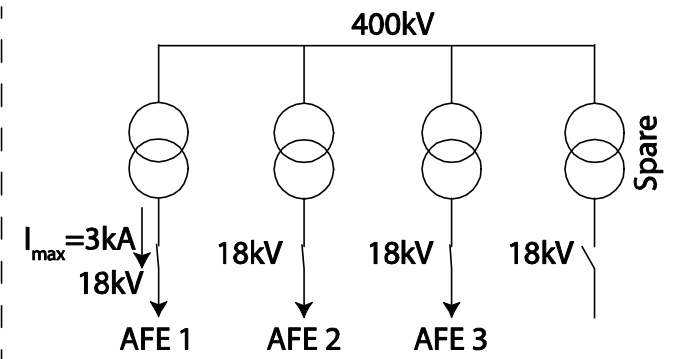

Fig. 3: Minimal transformers number possible distribution grid layouts for CLIC klystron modulators. a) with $66 \mathrm{kV}$ intermediate voltage bus-bar, and b) with direct step-down from $400 \mathrm{kv}$ to $18 \mathrm{kV}$. 
A certain amount of power fluctuation will be injected at the $18 \mathrm{kV}$ level. This will have the following consequences:

1. Voltage flicker at the $18 \mathrm{kV}, 66 \mathrm{kV}$ and $400 \mathrm{kV}$ voltage levels.

2. Power fluctuation which must be accepted by the grid provider and power producers.

The maximum and minimum three-phases short-circuit powers at the $400 \mathrm{kV}$ power lines at CERN are:

- $S_{\text {ccmax }}=14000 M V A$

- $S_{c c m i n}=3400 M V A$

These values correspond to the best and worst regional grid configuration [3] (number of operational producers in particular). The $400 \mathrm{kV}$ connection at CERN is considered to be very strong, and power fluctuation in the order of a few tens of MW is preliminarily considered not a problem since voltage flickers would be extremely low. However, studies are starting for deriving the implications on the power generators in terms of additional losses and mechanical vibrations (which at $50 \mathrm{~Hz}$ should be highly attenuated).

The voltage flicker amount on the $18 \mathrm{kV}$ and $66 \mathrm{kV}$ voltage levels depends on the short-circuit impedance of the distribution transformers. For instance hereafter are examples of existing power transformers at CERN:

- 110MVA (Yy0), 400kV/66kV: Short-circuit voltage $\mathrm{U}_{\mathrm{sc}}=11 \%$

- 70MVA (Yd11), 66kV/18kV: Short-circuit voltage $\mathrm{U}_{\mathrm{sc}}=8 \%$

- 90MVA (Yd11), 400kV/18kV: Short -circuit voltage $\mathrm{U}_{\mathrm{sc}}=14 \%$

Current fluctuations on the $18 \mathrm{kV}$ side will appear on the $66 \mathrm{kV}$ and $400 \mathrm{kV}$ in terms of voltage flickers, which amount depends on the transformer short-circuit impedance (or $\mathrm{U}_{\mathrm{sc}}$ ). Therefore it is sensible to dedicate a power network for the klystron modulators only in order to mitigate voltage flickering on other electrical equipment for CLIC (Magnets powering, services, etc.).

It is clear that a supplementary lower voltage level can be foreseen $(6 \mathrm{kV}$ or $3.3 \mathrm{kV}$ for example) in order to optimise the voltage adaptation to the AFEs.

\section{Cables Effect}

The MV cable parasitic inductance may be calculated for two simple cable types; two parallel round conductors and two coaxial conductors [4]. In the first case the inductance as calculated using (1) is plotted in Fig. 4(a).

$$
L=\frac{\mu_{o}}{4 \pi}+\frac{\mu_{o}}{\pi} \ln \frac{D}{\alpha}
$$




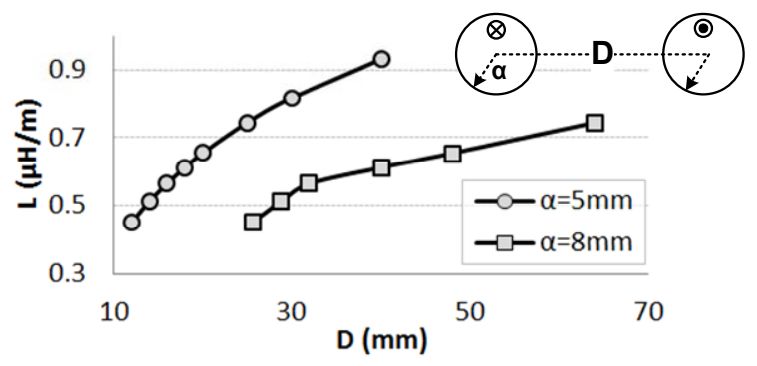

(a)

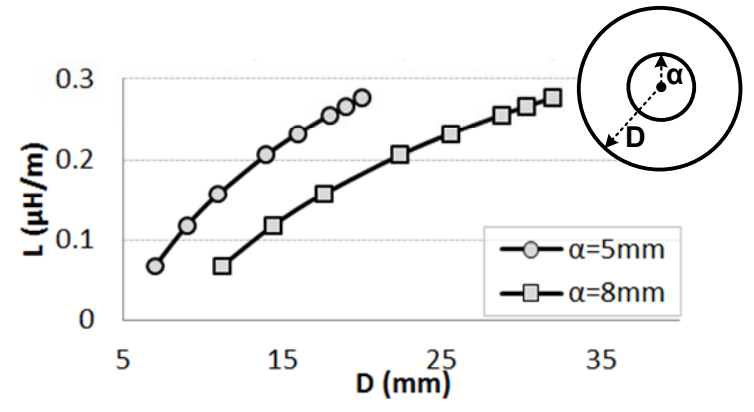

(b)

Fig. 4: MV cable inductance calculation in case of two (a) parallel running round conductors and (b) in case of two coaxial conductors

In case of two coaxial cables the inductance can be significantly lower as shown in Fig. 4(b). The importance of the parasitic becomes paramount in fault cases as well as transient operation.

$$
L=\frac{\mu_{o}}{2 \pi} \ln \left(\frac{D}{\alpha}\right)
$$

A number of cases in which cable parasitics become critical are illustrated in Fig. 5. The first case is short-circuit fault clearance that requires control of the inductive energy in cable loops in order to make it possible for breakers to open under normal voltage conditions. In reality, interrupting a DC current on the dc-side of the AFEs would be very difficult in a highly inductive circuit. The second case results in the loss of dynamic performance of the power system due to the cable parasitic inductances and capacitances effectively forming a filter for high frequency transients. Given the high dynamics required in this pulsed power application, cables may well-interfere with the circuit operation. And, finally, the parasitic components can be responsible for low frequency oscillations in the MVDC network in case of poor regulation performance.

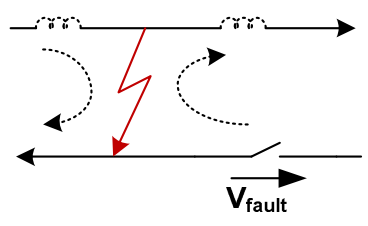

(a)

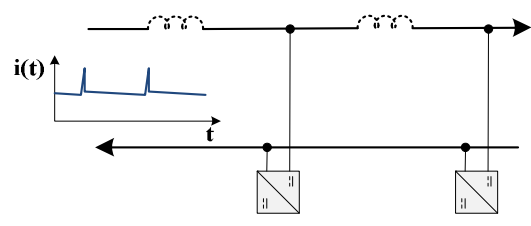

(b)

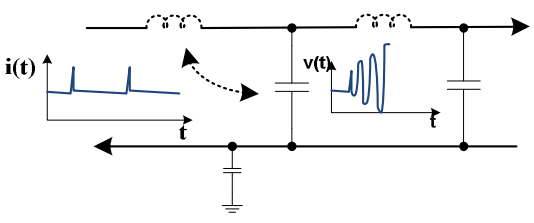

(c)

Fig. 5: Effects involving cable parasitic inductance; (a) short-circuit fault at random point (b) dynamic behaviour of pulsed power supplies and (c) oscillations caused between cable parasitic components during pulsed operation.

All of the above considerations make part of an normal and fault-condition analysis that needs to be performed for each candidate grid configuration.

\section{Active front-end design considerations}

The primary functionality of the AFE converter in this application is to perform the voltage regulation of the MVDC network. Using a voltage source converter is a good choice as they tend to achieve good voltage regulation with minimal harmonic impact on the grid side. In the recent years, multilevel converters have proven highly performing and more cost effective than standard 2-level solutions [5]. A number of topologies are being considered within the CLIC context. The multilevel families have certain advantages in the high voltage and high power range. In particular, the Modular Multilevel Converter (MMC) [6],[7],[8] has been identified as a potential choice for two main reasons; 
- Each converter module is a low voltage assembly made of standard industrial components which will allow many suppliers to be involved in the construction and operation. This aspect is particularly interesting for CERN.

- There are ways to introduce converter module redundancy in order to be able to bypass single points of failure and maintain normal operation under certain fault conditions.

\section{Voltage and current dynamics requirements}

As a Voltage Source Converter the DC side of an MMC is expected to be connected on to a stiff voltage supply. This is not the case in the present application. Fig. 6(a) illustrates the main power conversion chain from the utility grid to the output pulse to be applied to the klystron. To ensure a good dynamic response of the output voltage rise time (in Fig. 6 (c)) an energy storage capacitor bank is placed inside the klystron modulator, after the medium voltage HV (MVDC) line. Part of the energy storage can be placed in proximity to the AFE. The optimal sharing between energies storage elements $\left(C_{1}\right.$ and $C_{2}$ of Fig. 6(a)) depends on the MVDC line parameters and length. If the AFE is regulated with a constant voltage reference, then the associated current fluctuation results in a DC and AC power fluctuation. To avoid this, the multiplication between AFE's DC voltage and current must be constant (or nearly constant) at each time, leading to a voltage shape illustrated in Fig. 6(b) to be set as reference to the AFE [2]. Therefore, the ability of the AFE to draw constant power is directly linked to its ability to regulate fluctuating DC voltage and DC current. The AFE dynamic, combined with the amount of energy stored in $\mathrm{C} 1$ and $\mathrm{C} 2$, define the power system capability of reducing the power fluctuation experienced by the utility grid.

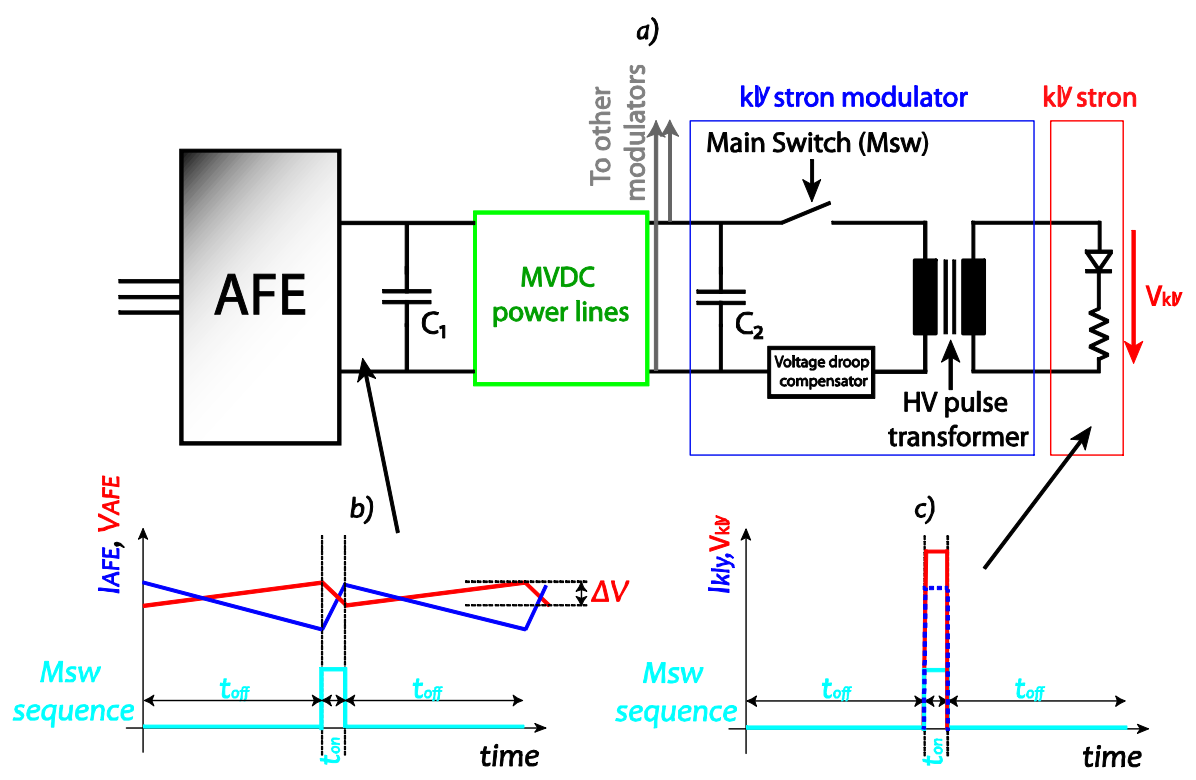

Fig. 6: a) Simplified conversion chain; b) AFE voltage and current shapes for constant power consumption; c) klystron modulator output pulse.

\section{Modular Multilevel Converter Design}

The modular multilevel converter comprises of a series-connected half bridge converter modules each of them containing a capacitor acting as a voltage source (Fig. 7). The operation is based the sequential insertion and bypass of the voltage sources in order to create the required sinusoidal voltages at the converter $\mathrm{AC}$ terminals.

It is evident that the topology includes an amount of internal energy storage that is (in DC operation) connected in parallel with any external DC link capacitance. The total module capacitance in a grid- 
connected MMC is typically around 20-40kJ/MVA. This implies that the DC link voltage may only fluctuate to the extent that it does not affect the converters capability to produce enough voltage on the AC terminals. The voltage difference between the dc-link and the sum of the module voltages will appear across the arm reactors and will generate a current ripple of the same frequency as the dc link voltage fluctuation.

There is a technical and economic evaluation to be performed in order to select the number of voltage levels (or arm modules) in an MMC. This involves all components (DC bank capacitors, cables, modulator dc-dc converter) connected to the AFE converter. In brief, raising the voltage brings the advantage of lower DC and AC current and hence lower power loss, less susceptibility to cable parasitic inductance, thinner cross-section and distance-immunity of connections. On the other hand a higher DC distribution voltage generally increases equipment costs; raises certain fault protection issues (such as the design of DC breakers) and introduces challenges to both the design and maintenance of such converters.

Several multilevel topologies exist. A number of solutions are being evaluated at CERN by its research partners. In this paper we focus on the MMC converter of Fig. 7, where each module is rated for the voltage $E$ (in an $\mathrm{N}_{\text {arm }}$-level bridge the module voltage rating is $2 E / N_{\text {arm }}$ ). As can be observed the MMC legs are connected in parallel to the DC-link which means that the total MMC leg voltage seen across all modules will be fluctuating with a frequency of $50 \mathrm{~Hz}$. It also implies that the reference waveform should be adjusted to account for DC bus variations or else the voltage difference across the arm reactors will be excessive.

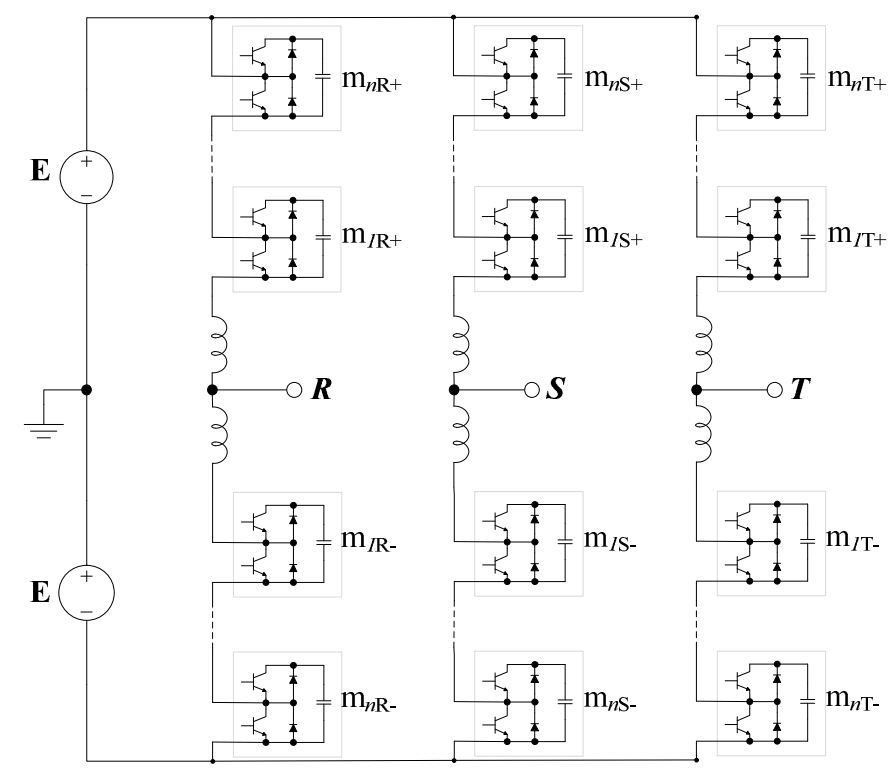

Fig. 7: The Modular Multilevel Converter schematic comprising of $n$ converter modules per arm, two arms per converter leg and three legs (for a three phase converter).

A first approach for finding a possible MMC configuration (number of levels, module capacitance) is based on the calculation of the relationship between the DC bus voltage level, the allowed module voltage fluctuation and the required energy storage per module. This calculation is based on the assumption that the sum of module capacitance voltages will not exceed a predetermined voltage ripple (to avoid the need for large arm reactors). 


\begin{tabular}{|c|c|c|c|c|c|c|c|}
\hline \multirow{2}{*}{$\begin{array}{c}\text { Nominal } \\
\text { DC bus } \\
\text { voltage }\end{array}$} & \multirow{2}{*}{$\begin{array}{c}\text { Arm } \\
\text { Current } \\
\text { (rms) }\end{array}$} & \multirow{2}{*}{$\begin{array}{c}\text { Arm } \\
\text { modules } \\
\left(\mathrm{N}_{\mathrm{arm}}\right)\end{array}$} & \multicolumn{5}{|c|}{ Module Capacitor Voltage Ripple: 5\% } \\
\hline & & & $\Delta \mathbf{V}$ & $\begin{array}{c}\text { module } \\
\text { capacitance }\end{array}$ & $\begin{array}{c}\text { Module capacitor } \\
\text { volume }\end{array}$ & $\begin{array}{c}\text { module } \\
\text { energy }\end{array}$ & $\begin{array}{c}\text { Arm } \\
\text { energy }\end{array}$ \\
\hline $10 \mathrm{kV}$ & $25.4 \mathrm{kA}$ & 9 & $0.5 \mathrm{kV}$ & $1170 \mathrm{mF}$ & $5.87 \mathrm{~m}^{3}$ & $725 \mathrm{~kJ}$ & $6.52 \mathrm{MJ}$ \\
\hline $20 \mathrm{kV}$ & $12.7 \mathrm{kA}$ & 17 & $1 \mathrm{kV}$ & $555 \mathrm{mF}$ & $2.77 \mathrm{~m}^{3}$ & $384 \mathrm{~kJ}$ & $6.52 \mathrm{MJ}$ \\
\hline $25 \mathrm{kV}$ & $10.1 \mathrm{kA}$ & 21 & $1.25 \mathrm{kV}$ & $438 \mathrm{mF}$ & $2.19 \mathrm{~m}^{3}$ & $311 \mathrm{~kJ}$ & $6.52 \mathrm{MJ}$ \\
\hline $100 \mathrm{kV}$ & $2.5 \mathrm{kA}$ & 84 & $5 \mathrm{kV}$ & $110 \mathrm{mF}$ & $0.55 \mathrm{~m}^{3}$ & $77.7 \mathrm{~kJ}$ & $6.52 \mathrm{MJ}$ \\
\hline $150 \mathrm{kV}$ & $1.7 \mathrm{kA}$ & 125 & $7.5 \mathrm{kV}$ & $72 \mathrm{mF}$ & $0.36 \mathrm{~m}^{3}$ & $52 \mathrm{~kJ}$ & $6.52 \mathrm{MJ}$ \\
\hline $200 \mathrm{kV}$ & $1.3 \mathrm{kA}$ & 167 & $10 \mathrm{kV}$ & $54 \mathrm{mF}$ & $0.27 \mathrm{~m}^{3}$ & $39.1 \mathrm{~kJ}$ & $6.52 \mathrm{MJ}$ \\
\hline
\end{tabular}

Table I: Calculations of the number of arm modules and associated energy storage per module for various DC-link voltage levels and for a capacitor voltage ripple of 5\%

Table I summarises the effect of different nominal DC bus voltages on the MMC design. Clearly reducing the dc-bus voltage below $100 \mathrm{kV}$ will introduce challenges in finding suitable semiconductors to handle the resulting arm current (note that the switch rms current is somewhat lower than the arm current due to the pwm operation). Additionally, when low voltage is used and smaller number of arm modules $\mathrm{N}_{\text {arm }}$ will normally be employed and consequently larger module capacitance will be needed to keep the module capacitance voltage ripple below 5\%. As $\mathrm{N}_{\text {arm }}$ increases the energy storage needed per module decreases. Capacitive energy storage up to $40 \mathrm{~kJ}$ is feasible in terms of mechanical integration of the converter. Given the aforementioned constraints it becomes clear that dc-side voltages of less than $150 \mathrm{kV}$ will not be suitable unless the number of arm modules and perhaps phase legs are increased in order to reduce the module capacitance and reduce the nominal arm current.

\begin{tabular}{|c|c|c|c|c|c|c|c|}
\hline \multirow{2}{*}{$\begin{array}{l}\text { Nominal } \\
\text { DC bus } \\
\text { voltage }\end{array}$} & \multirow{2}{*}{$\begin{array}{c}\text { Arm } \\
\text { Current } \\
\text { (rms) }\end{array}$} & \multirow{2}{*}{$\begin{array}{c}\text { Arm } \\
\text { modules } \\
\left(\mathrm{N}_{\mathrm{arm}}\right)\end{array}$} & \multicolumn{5}{|c|}{ Module Capacitor Voltage Ripple:: $10 \%$} \\
\hline & & & $\Delta \mathbf{v}$ & $\begin{array}{c}\text { module } \\
\text { capacitance }\end{array}$ & $\begin{array}{c}\text { Module capacitor } \\
\text { volume }\end{array}$ & $\begin{array}{l}\text { module } \\
\text { energy }\end{array}$ & $\begin{array}{c}\text { Arm } \\
\text { energy }\end{array}$ \\
\hline $10 \mathrm{kV}$ & $25.4 \mathrm{kA}$ & 9 & $1 \mathrm{kV}$ & $587 \mathrm{mF}$ & $2.9 \mathrm{~m}^{3}$ & $362 \mathrm{~kJ}$ & $3.26 \mathrm{M}$ \\
\hline $20 \mathrm{kV}$ & $12.7 \mathrm{kA}$ & 17 & $2 \mathrm{kV}$ & $277 \mathrm{mF}$ & $1.4 \mathrm{~m}^{3}$ & $192 \mathrm{~kJ}$ & $3.26 \mathrm{M}$ \\
\hline $25 \mathrm{kV}$ & $10.1 \mathrm{kA}$ & 21 & $2.5 \mathrm{kV}$ & $220 \mathrm{mF}$ & $1.1 \mathrm{~m}^{3}$ & $155 \mathrm{~kJ}$ & $3.26 \mathrm{M}$ \\
\hline $100 \mathrm{kV}$ & $2.5 \mathrm{kA}$ & 84 & $10 \mathrm{kV}$ & $54.8 \mathrm{mF}$ & $0.27 \mathrm{~m}^{3}$ & $38.8 \mathrm{~kJ}$ & $3.26 \mathrm{M}$ \\
\hline $150 \mathrm{kV}$ & $1.7 \mathrm{kA}$ & 125 & $15 \mathrm{kV}$ & $36.3 \mathrm{mF}$ & $0.18 \mathrm{~m}^{3}$ & $26.1 \mathrm{~kJ}$ & $3.26 \mathrm{M}$ \\
\hline $200 \mathrm{kV}$ & $1.3 \mathrm{kA}$ & 167 & $20 \mathrm{kV}$ & $27.2 \mathrm{mF}$ & $0.14 \mathrm{~m}^{3}$ & $19.5 \mathrm{~kJ}$ & $3.26 \mathrm{M}$ \\
\hline
\end{tabular}

Table II: Calculations of the number of arm modules and associated energy storage per module for various DC-link voltages and for a capacitor voltage ripple of $10 \%$

Table II summarises the preliminary design calculations of an MMC with $10 \%$ ripple on the module capacitor voltages. The conclusion that can be drawn, if compared with the results of Table I, is that allowing a higher voltage ripple relaxes the energy storage requirements at module level, hence allowing a lower overall dc link voltage to be employed.

The energy storage per module (which is directly proportional to the capacitor volume) decreases exponentially as total DC link voltage increases (Fig. 8) whereas in all cases the total stored energy in the MMC modules is independent of the dc link voltage, as long as the total converter rated power remains unchanged.

The current flowing through each leg will determine the silicon area of the semiconductors used to build the converter modules. The leg current comprises of two components; the dc current component and the load AC current. Both currents decrease proportionally as the dc link voltage decreases (Fig. $8)$. 

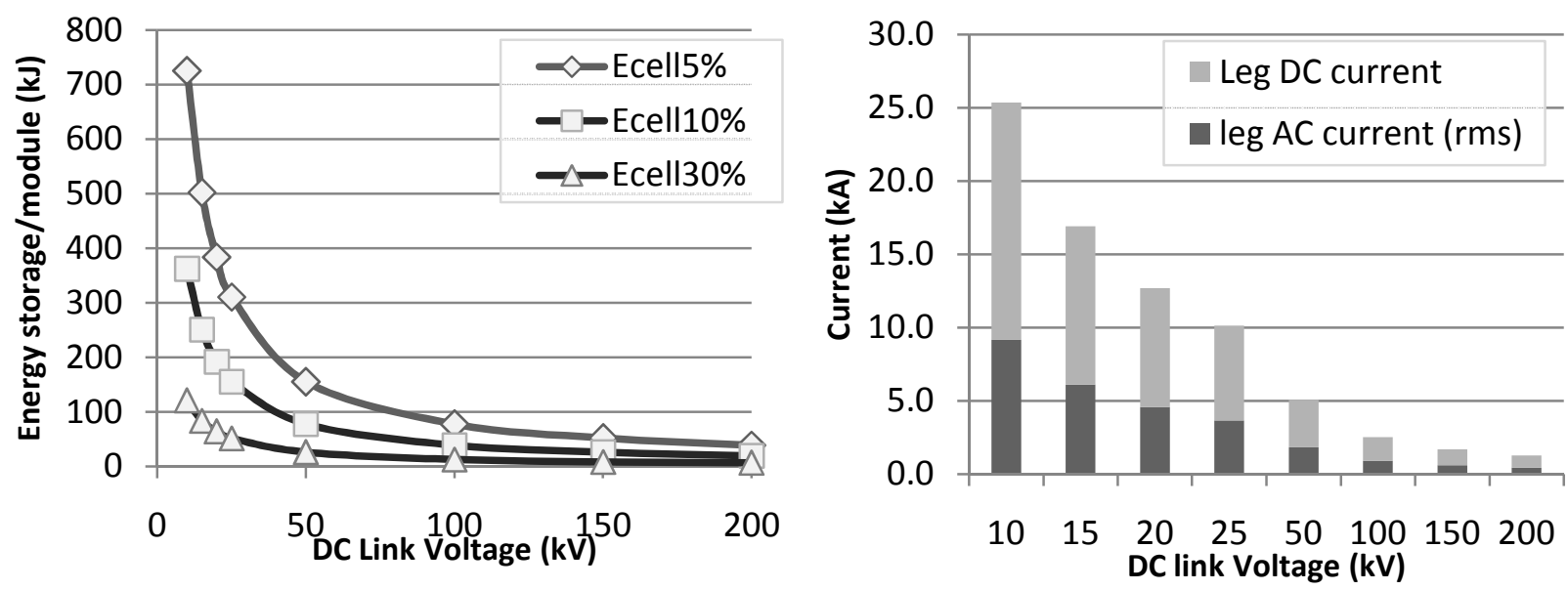

Fig. 8: Energy stored per module with respect to DC link voltage (left) and the rms current through each converter leg (right) assuming a module voltage of $1200 \mathrm{~V}$.

\section{Conclusion}

This paper reports on the progress made in studying the CLIC Power System layout. Defining the DC distribution grid is critical in several ways such as the choice of protection of equipment, sizing and positioning of capacitive energy storage and finally interfacing to the external power grid. A list of the most critical design issues is drawn, some trends are presented regarding the DC bus voltage selection and finally an assessment of the MMC topology in this pulsed power application is proposed. Preliminary assessment indicates that grouping of the loads in three to several Active Front-end Converters could advantageous. Additionally, if a high voltage multilevel converter is employed to provide the bulk of power, a minimum voltage in the order of $100 \mathrm{kV}$ is needed to limit the current ratings of components and keep energy storage of modules within a reasonable volume.

\section{References}

[1] Aguglia D., Martins C. A., Cerqueira Bastos M., Nisbet D., Siemaszko D., Sklavounou E., Viarouge P., "Klystron Modulator Technology Challenges for the Compact Linear Collider (CLIC) ", in proc., IEEE Conference on Pulsed Power, in IEEE Proc. Pulsed Power Conference (PPC), Washington DC, 2011, pp. 1413- 1421.

[2] Aguglia D., Sklavounou E., "Klystron Modulators Capacitor Chargers Design Compromises for AC Power Quality Increase of the Compact Linear Collider (CLIC) ", in Proc. Int. Symp. On Power Elect., Elect. Drives, Automation and Control (SPEEDAM), Sorrento (IT), 2012, pp. 1535 - 1541.

[3] Kahle, K., Calculation of fault currents in the CERN power distribution network, CERN technical note, EDMS no. 853192/4, July 2007, pp. 1- 109

[4] Leferink, F. B. J., "Inductance calculations; Methods and equations," in IEEE Proc. International Symposium on Electromagnetic Compatibility, 1995, pp. $16-22$.

[5] Papastergiou, K.D. Wheeler, P.W. Clare, J.C., "Comparison of losses in multilevel converters for aerospace applications", Power Electronics Specialists Conferenced (PESC), Rodos, Greece, 2008

[6] Lesnicar A. and Marquardt R., "A new modular voltage source inverter topology," European Power Electronics Conference (EPE), Toulouse, France, September 2-4, 2003

[7] Glinka M. and Marquardt R., "A new ac/ac multilevel converter family," IEEE Transactions on Industrial Electronics, vol. 52, no. 3, 2005

[8], P. Asimakopoulos, MSc Thesis: "Design and Control of a Modular Multilevel Converter in an Active Frontend Application", Chalmers University of Technology, 2013 